\title{
Quantified and Perceived Unevenness of Solid Printed Areas
}

\author{
Albert Sadovnikov, Lasse Lensu, \\ Joni-Kristian Kamarainen, and Heikki Kälviäinen \\ Laboratory of Information Processing, Department of Information Technology, \\ Lappeenranta University of Technology, P.O.Box 20, 53851 Lappeenranta, Finland \\ \{sadovnik, ltl, jkamarai, kalviai\}@lut.fi
}

\begin{abstract}
Mottling is one of the most severe printing defects in modern offset printing using coated papers. It can be defined as undesired unevenness in perceived print density. In our studies, we have implemented two methods known from the literature to quantify print mottle: the standard method for prints from office equipment and the bandpass method specially designed for mottling. Our goal was to study the performance of the methods when compared to human perception. For comparisons, we used a test set of 20 grey samples which were assessed by professional and non-professional people, and the artificial methods. The results show that the bandpass method can be used to quantify mottling of grey samples with a reasonable accuracy. However, we propose a modification to the bandpass method. The enhanced bandpass method utilizes a contrast sensitivity function for the human visual system directly in the frequency domain and the function parameters are optimized based on the human assessment. This results a significant improvement in the correlation to human assessment when compared to the original bandpass method.
\end{abstract}

\section{Introduction}

Print quality is an essential attribute when modern printing processes are considered. This is because an increasing proportion of data to be printed are images. If the original of a print is assumed to be ideal, print quality depends on the printability of paper, printing inks, and printing process. Despite major improvements in the before-mentioned factors affecting the quality, there are several undesired effects in prints. One of the most severe defects is mottling which is the uneven appearance of solid printed areas. It is related to density and gloss of print, and it is caused by non-ideal interactions of paper and ink in high-speed printing processes. There are three types of mottling depending on the cause for this defect: back-trap mottle, water-interface mottle, and ink-trap mottle. The causes for these forms of mottling are uneven ink absorption in the paper, insufficient and uneven water absorption of the paper, and incorrect trapping of the ink because of tack, respectively [1. However, a thorough explanation to this phenomenon is still missing. 
Mottling can be defined as undesired unevenness in perceived print density, or more technically as "aperiodic fluctuations of density at a spatial frequency less than 0.4 cycles per millimeter in all directions" 2. When printing defects are of concern, mottling is generally considered as a stochastic phenomenon. Depending on the cause, however, print unevenness can include several forms of regularity. For example, a regular drift in the printing process causes macro-scale noise in print, whereas structures in the paper formation are random in nature and cause micro-scale noise.

A few methods to quantify mottling by a machine vision system have been proposed. The ISO 13660 standard includes a method for monochrome images. The method is based on computing the standard deviation of small tiles within a larger area [2]. In the standard, the size of the tiles is set to a fixed value, which is a known limitation [3. The standard method has been improved by using tiles of variable sizes [4. Other methods relying on clustering, statistics, and wavelets have also been proposed to quantify mottling [5617]. Other approaches to evaluate greyscale mottling have their basis in frequency-domain filtering $[8$, and frequency analysis $[9$. All of the before-mentioned methods are designed for binary or greyscale images. If colour prints were assessed, the performance of the methods would be limited when compared to human assessments.

It is possible to define mottling by using mathematical or physical terms. However, mottling is implicitly related to human perception: If a person looking at a solid print perceives unevenness, mottling is considered as a defect. Thus, a strict definition based on the quantitative sciences can prove to be insufficient. This is why the properties and limits of the human visual system (HVS) must be taken into account in the design of proper methods to quantify mottling. Sensitivity of the HVS to contrast and spatial frequencies of noise in images is independent of luminance within common luminance levels [10]. However, the contrast sensitivity depends on the spatial frequency [11, thus, mottles of different sizes are perceived differently. The peak sensitivity of the HVS is approximately at 3 cycles/degree, and the maximum detected frequency is from 40 cycles/degree (sinusoidal gratings) [12] to over 100 cycles/degree (single cycle) [13.

The purpose of our work was to implement study artificial methods to quantify mottling, and compare the method results to evaluations by humans. Since the grounds of the selected methods are not directly in vision science, we propose a modification to the method which is superior based on the comparison. The modification is in accordance with the psychophysical studies in vision science, and it utilizes the frequency information of the sample images directly.

\section{Methods}

To study the possibilities of machine vision, we implemented two methods to quantify print mottle: the standard method to assess image quality of printer systems [2], and the bandpass method [8]. The third method described in this work is a modification of the bandpass method accommodating an appropriate contrast sensitivity function (CSF) for the HVS. 


\subsection{Standard Method}

ISO 13660 standard is designed for assessing print quality of office equipment that produce monochrome prints [2]. The attributes of print density for large print areas include graininess and mottling. In the standard, a fixed value has been chosen to separate this two forms of print unevenness. Aperiodic fluctuations of print density at spatial frequencies higher than 0.4 cycles/degree are considered as graininess, whereas frequencies lower than 0.4 cycles/degree are mottling. The standard method is presented in Algorithm 1 .

\section{Algorithm 1 Standard method}

1: Divide the region of interest into tiles.

2: Compute the mean densities within each tile.

3: Compute the standard deviation of the means as the measure of mottling.

The region of interest must be larger than $21.2 \mathrm{~mm}$ squared, and it is divided into tiles of size $1.27 \mathrm{~mm}$ squared. Within each tile, 900 independent measurements of density are made.

\subsection{Bandpass Method}

The method is based on applying a set of Gaussian bandpass filters to the image in the frequency domain. The coefficient of variation of reflectance $\left(\mathrm{CV}_{R}\right)$ for each spatial image representing a frequency band is computed. Different coefficients represent the variation of reflectance within each band [8]. The coefficients are weighted with the CSF and then summed together as the mottling index. The method is described in Algorithm 2 .

\section{Algorithm 2 Bandpass method}

1: Filter the image with a set of bandpass filters.

2: Compute coefficients of variation from the filtered spatial image for each frequency band.

3: Weight each coefficient with a CSF.

4: Sum the weighted coefficients to get the mottling index.

In Step 1, the image is filtered in the frequency domain with a set of bandpass filters. Five fixed spatial bands are designed to an octave series: $0.5-1,1-2,2-4$, 4-8, and 8-16 $\mathrm{mm}$ (note that we fixed the viewing distance to $30 \mathrm{~cm}$ in the human assessments). The band containing the smallest details has been included when compared to 8]. The Gaussian filters are illustrated in Fig. 11 The mean (DC component) is set to 1 so that the mean grey value of the image does not change due to filtering.

In Step 2, the coefficients of variation for each band are computed in the spatial domain. The coefficient of variation is the ratio of standard deviation of reflectance and mean reflectance, i.e.,

$$
\mathrm{CV}_{R}=\frac{\sigma_{R}}{R}
$$




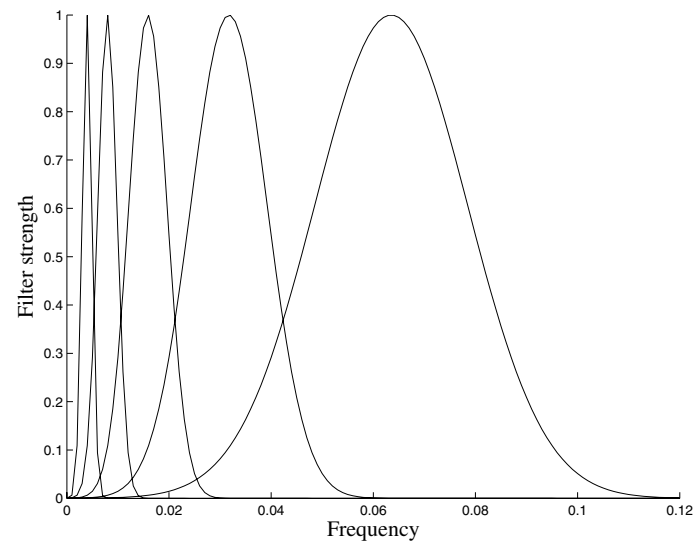

Fig. 1. The filters in 2-D representing the $0.5-1,1-2,2-4,4-8$, and 8-16 mm spatial bands

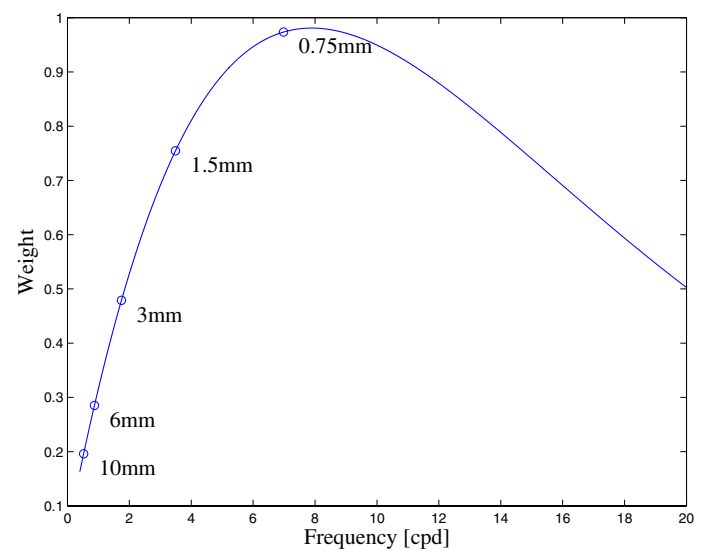

Fig. 2. The Mannos monochrome CSF and the weights corresponding to $0.75,1,5,3$, 6 , and $10 \mathrm{~mm}$

In Step 3, the coefficients are weighted with a CSF 14 illustrated in Fig. 2. The weights are taken at points representing $0.75,1.5,3,6$, and $10 \mathrm{~mm}$.

\subsection{Enhanced Method}

The idea for this method comes from the bandpass method. Consider a peak in frequency domain which lies in between two bandpass filters, it introduces serious sinusoidal distortion (unevenness) in spatial domain. At the same time, it cannot be detected by a predefined set of bandpass filters, and thus the computed mottling index remains intact. The obvious solution to this bandpass method 
weakness is to increase number of bandpass filters in order to catch more frequency fluctuations. Finally, increase in the number of filters leads to a limit case, where all the bandpass filters comprise a plane, which has no impact on the values of frequency magnitudes. However, coefficient of variation used as weighted value, makes it complicated for integration in the limit case. We propose to use the following value

$$
\overline{\mathrm{CV}}_{R}=\frac{\sigma_{R}^{2}}{R}
$$

to make the result correspond to the bandpass method in the terms of order of magnitude, we take square root inside the integral. In the limit case mottling index will have the form (follows from Parseval's theorem)

$$
M=\frac{1}{F(0,0)} \int_{-\infty}^{\infty} \int_{-\infty}^{\infty} C S F(u, v) \sqrt{F(u, v) \bar{F}(u, v)} d u d v,
$$

where $F(0,0)=R$ is the mean reflectance, $\operatorname{CSF}(u, v)$ is a 2-D representation of the CSF, $F$ denotes the Fourier transform of the image, and $\bar{F}$ is the complex conjugate of the transformed image.

Since the frequency for the peak contrast sensitivity of the HVS varies from $2 \mathrm{cpd}$ up to $10 \mathrm{cpd}$, depending on the type of gratings and its regularity [10], it was decided to introduce the scaling factor into the CSF formulation. The factor scales the function along the frequency axis. It was experimentally found that, for the unevenness type gratings, the peak sensitivity is approximately at $2 \mathrm{cpd}$. This can be explained by the stochastic nature of mottling and its aperiodicity.

We also studied the effect of orientation sensitivity of the HVS [12. It is known that human sensitivity is lowest around $45^{\circ}$ and $135^{\circ}$ and highest at vertical and horizonal directions. However, experiments showed low significance of introducing the orientational scaling. This can also be understood based on the nature of mottling.

\subsection{Visual Assessment}

To compare the results of the implemented methods to human perception, we collected a set of 20 mottling samples covering a wide range of mottling, and asked human observers to evaluate the perceived mottling. The group of observers consisted of experts from the paper industry, and "laymen" in the area of image processing. The mean of these subjective assessments was used as an initial ground truth for mottling, and the results of all the machine vision methods were compared to this information.

The human assessment consisted of two parts. The first part was a pairwise evaluation of the whole sample set: the observer was asked to select the sample which had less mottling. The main function of this part was to present all samples to the observer, and to give some idea of different forms and levels of mottling. In the second part, each sample was evaluated one at a time, and the observer was asked to rate the level of mottling in a five point Likert scale. Two control 
questions were used in the assessment: the number of times the person had evaluated mottling, and the time needed for the test. The primary function of the assessment was to quantify the perceived level of mottling of the test set.

The results of the assessments were processed as follows. The people taking the test were divided into two distinct groups based on the control question about the number of times the person had evaluated mottling. The first group was formed by experts who evaluate prints as a part of their work. The second group consisted of people who evaluated mottling for the first time and were not experts in the field of print assessment. Selection criteria for outliers were difficult to design. Each observer had his or her own way of selecting the the use of the scale. The mean and the standard deviation were used as elementary criteria to select outliers. If either one differed from the average of all assessments significantly, the assessment was marked as an outlier.

\section{Experiments}

We present the results for the set of $20 \mathrm{~K} 70$ (70\% black) samples (see Fig 3 ). The original samples are approximately $4.5 \mathrm{~cm} \times 4.5 \mathrm{~cm}$ in size. The paper used for printning is $70 \mathrm{~g} / \mathrm{m}^{2}$ LWC (Lightweight Coated) paper, and the samples were printed using heatset offset printing process with round dots. The samples were originally scanned with a flatbed office scanner at 1200 dpi and gamma of 2.2. The gamma value was not altered before applying the machine vision methods. To reduce computing time, the images were re-sampled to 600 dpi because this resolution is more than sufficient in this application when the HVS is concerned.

We inspected mottle sizes ranging from 0.5 to $16 \mathrm{~mm}$ while viewing the sample from a distance of $30 \mathrm{~cm}$ (spatial frequency range 0.03-1 cycles $/ \mathrm{mm}$ ). Spatially higher- and lower-frequency unevennesses were considered as graininess and banding. The viewing angle of all samples was approximately $8.5^{\circ}$, and the material surrounding each sample was $100 \%$ black cardboard. To remove possible defects in images that are not mottling, the inspected contrast of print density was limited to $\pm 10 \%$ of the median grey-value of an image.

\subsection{Visual Assessment}

The results are based on 35 human evaluations. The assessments were made in usual office lighting conditions. However, the conditions were not identical in all evaluations, thus, the human assessment should be considered as an initial one. Evaluators were divided into two groups: experts (12 people) and laymen (23 people). The division was made based on the number of mottling evaluations done prior to this one. As it can be seen from Fig. 4(a) there is only little difference in evaluations between the experts and laymen. This is natural since it would be confusing if the experts evaluated print quality of samples in which mottling is most visible completely distinctly to end-users. However, experts in the printing industry do have a different view of print quality, and there should be representatives from this group in the next and more thorough assessment. 

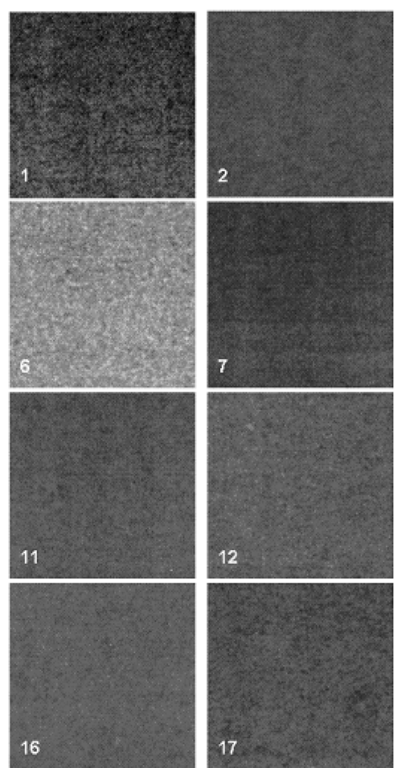
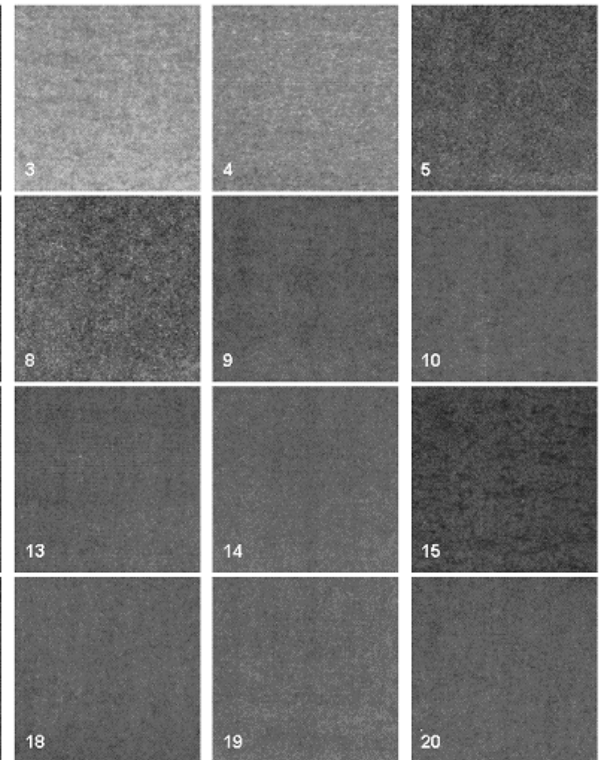

Fig. 3. The set of K70 samples (scaled to fit the figure and altered to enhance the visibility of mottling)

Confidence bounds in Fig. 4(a) show the average results across the whole population \pm standard deviation, and show how similar the mottling indices were among all evaluators.

\subsection{Machine Vision Assessment}

The standard method was implemented as described in the ISO 13660 standard [2]. The implementation of this method is easy and does not require much programming effort. As it was expected, the results produced by the standard method show low correlation to the human assessment (see Fig. 4(b)). In the standard, the size of the tiles is set to a fixed value which is a known limitation 3 . The bandpass method makes use of a few frequency bands to separate information relevant to the HVS. A small number of bands limits the number of spatial classes, and the method becomes similar to a set of low-pass filters used in previous mottling methods. Performance of the method is limited by the resolution of the image and the number of bands. The results of this method can be seen in Fig. 4(c), The increase in the number of bands leads to the enhanced method, which utilizes characteristics of the HVS and outperforms two aforementioned methods (see Fig. 4(d)).

All the artificial methods produced mottling indexes in their own scale. Thus, appropriate scaling was needed for the method comparison. We used simple 


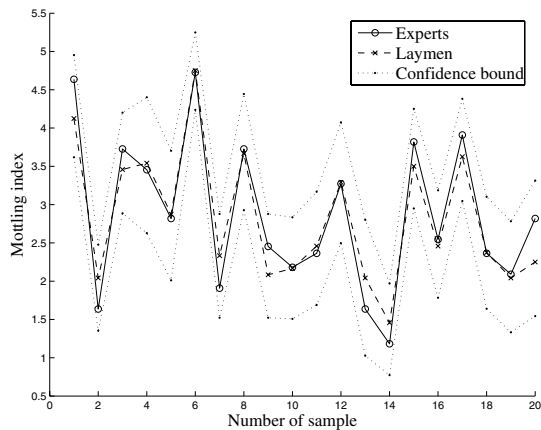

(a)

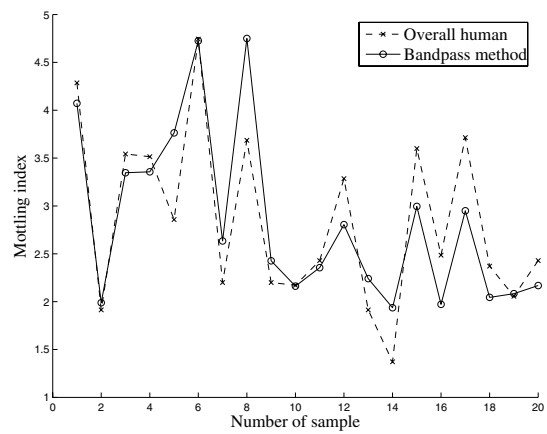

(c)

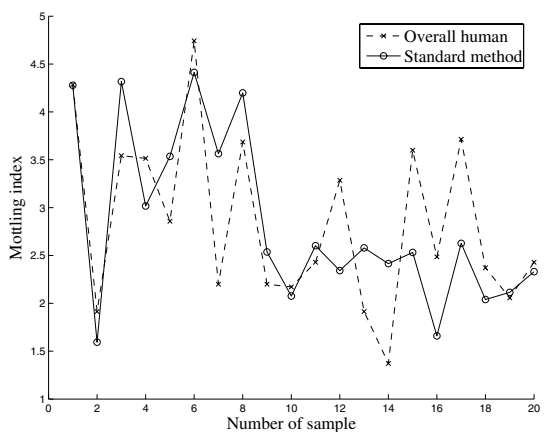

(b)

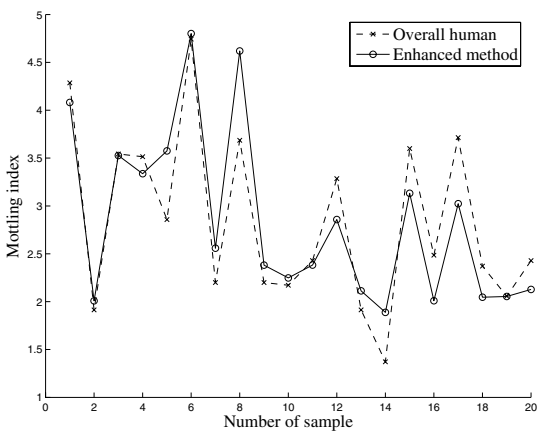

(d)

Fig. 4. Mottling assessments: (a) Human evaluation; (b) Standard method; (c) Bandpass method; (d) Enhanced method

normalization which equalizes the mean value and standard deviation of the experimental values across the samples.

\subsection{Results Summary}

In Table 1, inter-method similarity is presented. Correlation coefficients were used as the similarity measure.

Table 1. Mottling assessment correlations

\begin{tabular}{|l|c|c|c|c|c|c|}
\hline Methods & Overall & Experts & Laymen & Standard & Bandpass & Enhanced \\
\hline Overall human & 1.0000 & 0.9848 & 0.9957 & 0.6956 & 0.8579 & 0.8941 \\
Experts & 0.9848 & 1.0000 & 0.9644 & 0.6568 & 0.8125 & 0.8516 \\
Laymen & 0.9957 & 0.9644 & 1.0000 & 0.7078 & 0.8715 & 0.9057 \\
Standard & 0.6956 & 0.6568 & 0.7078 & 1.0000 & 0.8810 & 0.8755 \\
Bandpass & 0.8579 & 0.8125 & 0.8715 & 0.8810 & 1.0000 & 0.9949 \\
Enhanced & 0.8941 & 0.8516 & 0.9057 & 0.8755 & 0.9949 & 1.0000 \\
\hline
\end{tabular}


Fig. 4 shows performance graphs for different assessment approaches.

The collected correlation data allow to state that the enhanced method outperforms the other two methods. It can be also noticed that the machine vision methods correlate better among each other than with human evaluation based data. This leads to the conclusion that all artificial methods have a similar nature and the model of HVS they assume is not accurate.

\section{Conclusions}

In the presented work, we performed a comparison between the human and machine vision evaluation of mottling. The results of the human evaluation appear to be highly distributed and, thus, a larger number of assessments is needed both in evaluators and in samples. The high deviation in single sample evaluation results leads to the conclusion that a machine vision system modelling an average end-user is necessary. This could bring more precision in delivering printed products of desired quality.

The presented machine vision methods, though having a relatively good correlation with averaged human observation, still need improvement in the sense of modelling of the HVS. The standard method presented can be considered only as a starting point because this method does not model the HVS at all and also it does not have significant correlation with the human mottling evaluation. The bandpass method shows good results, though it should be mentioned, that it is not accurate to use CSF derived for regular sinusoidal gratings for measuring human sensitivity for random reflectance fluctuations. General enhancement for the bandpass method resulted improvement in both computational sense and in precision.

The goals for the future research can be defined as follows: make methods closer to human perception, by involving new knowledge about the HVS, and incorporate mottling evaluation of colour samples. The general conclusion of our research, is that for the implementation of a machine vision solution to the human perception problem, one needs a suitable HVS model and good statistical characteristics of how the humans perceive the phenomenon.

However, the results also show that when assessing low-contrast unevenness of print, humans have diverse opinions about quality.

\section{Acknowledgments}

This work was done as a part of Papvision project funded by European Union, National Technology Agency of Finland (TEKES Projects No. 70049/03 and 70056/04), and Academy of Finland (Project No. 204708).

\section{References}

1. IGT information leaflet w57: Back trap mottle. WWW:www.igt.nl (2002) [Accessed 2005-02-25]. Available: http://www.igt.nl/igt-site-220105/index-us/ w-bladen/GST/W57.pdf. 
2. ISO/IEC 13660:2001(e) standard. information technology - office equipment - measurement of image quality attributes for hardcopy output - binary monochrome text and graphic images. ISO/IEC (2001)

3. Briggs, J., Forrest, D., Klein, A., Tse, M.K.: Living with ISO-13660: Pleasures and perils. In: IS\&Ts NIP 15: 1999 International Conference on Digital Printing Technologies, IS\&T, Springfield VA (1999) 421-425

4. Wolin, D.: Enhanced mottle measurement. In: PICS 2002: IS\&T's PICS conference, IS\&T (2002) 148-151

5. Armel, D., Wise, J.: An analytic method for quantifying mottle - part 1. Flexo (1998) 70-79

6. Armel, D., Wise, J.: An analytic method for quantifying mottle - part 2. Flexo (1999) 38-43

7. Streckel, B., Steuernagel, B., Falkenhagen, E., Jung, E.: Objective print quality measurements using a scanner and a digital camera. In: DPP 2003: IS\&T International Conference on Digital Production Printing and Industrial Applications. (2003) 145-147

8. Johansson, P.Å.: Optical Homogeniety of Prints. PhD thesis, Kunglika Tekniska Högskolan, Stockholm (1999)

9. Rosenberger, R.R.: Stochastic frequency distribution analysis as applied to ink jet print mottle measurement. In: IS\&Ts NIP 17: 2001 International Conference on Digital Printing Technologies, IS\&T, Springfield VA (2001) 808-812

10. Barten, P.: Contrast Sensitivity of the Human Eye and its Effects on Image Quality. SPIE (1999)

11. Schade, O.H.: Optical and photoelectric analog of the eye. Journal of the Optical Society of America 46 (1956) 721-739

12. Kang, H.R.: Digital Color Halftoning. SPIE \& IEEE Press (1999)

13. Campbell, F.W., Carpenter, R.H.S., Levinson, J.Z.: Visibility of aperiodic patterns compared with that of sinusoidal gratings. Journal of Physiology (204) 283-298

14. Mannos, J., Sakrison, D.: The effects of a visual fidelity criterion on the encoding of images. IEEE Transactions on Information Theory 20 (1974) 525-536 